\title{
Abstracts for Posters
}

P27

\section{The RSLE Sequence in the Cytoplasmic Domain of Cell Adhesion Molecule L1 Is Involved in A Determinant Step of Cell Migration}

Hiroaki Asou" ", Kovichi Itoh" and Keiichi Uyemura"

1)Depertment of Neuro-cell bialogy, Tokyo Metropolitan Institute of Gerontology,Sakaecho,Itabashi-ku, Tokyo 173,Japan

2)The Tokyo metropolitan Institute of Medical Science,Honkomagome, Bunkyo-ku

Tokyo 113,Japan

3)Department of Physiology, Keio University, School of Medicine

Shinanomachi,Shinjuku-ku, Tokyo160,Japan

L1 is alternatively spliced exon which codes for part of the extreme-N-terminus region, and for the four amino acids Arg-Ser-Leu-Glu (RSLE) in the cytoplasmic region (1). To investigate functional differences of $\mathrm{L} 1$ variant, we have established respective cell lines transfected with either a cDNA lacking the cytoplasm $12 \mathrm{bp}$ (corresponding to amino acids-RSLE) (L4-2 cell) or complete / full-length CDNA (L3-1 cell). Western blot analysis revealed that the expression levels of L1 in L3-1 cells and L4-2 cells demonstrated similar levels of adhesion competence. Migratory ability of L3-1 cells from aggregates on to monolayers of $\mathrm{L} 1$ expressing cells, however, was found to be much more prominant than that of L4-2 cells. Similary, when $L 1$ fragments were used as a substrate, L3-1 cells migrated over larger areas of the dish compared to L4-2 cells, even through, both cell types were capable of migration over other substrates (i.e. PLL plus fibronectin, PLL plus laminin). After the process of cell migration, the serine residues of L1 fragment from L3-1 cells were markedly phosphorylated compared to those of L4-2. These results suggest that L4-2 cells differ from L3-1 cells in the ability to promote migration. We conclude that this is caused by : defference in concluded by the RSLE sequence and by protein serine phosphorylation mediated via homophilic interaction such as L1-L1.

(1)Miura, M. ,Asou, H. ,Kobayashi, M. and Uyenura, K. :Functional expression of a fulllength $\mathrm{cDNA}$ coding for rat neural cell adhesion molecule 11 mediates homophilic intercellular adhesion and migration of cerebellar neurons, J. Biol.Chem, 267, 1075210758. 


\title{
Abstracts for Posters
}

\section{P28}

\section{Oligodendrocyte maturation in vitro occures in a number of discrete stages.}

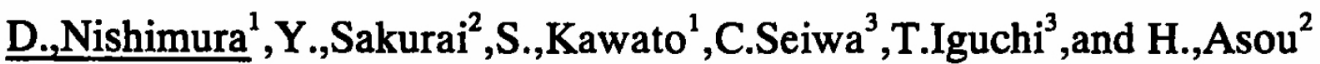 \\ Dept.of Biophysics and Lifescience,Univ.of Tokyo ${ }^{1}$, \\ Dept.of Cell Biology,TMIG ${ }^{2}$,Graduate School of Integrated Science, \\ Yokohama City Univ. ${ }^{3}$
}

We report mass-production technique that allow one to develop and maintain cultures stage-specific oligodendrocytes from embryonic rat brain. After four weeks cultures, they consist of more than $99 \%$ oligodendrocytes and fewer than 1\% non-oligodendroglial cells. When the oligodendrocyte lineage-specific glycolipid antibodies A2B5, GC, O4 and O1 were used to identify the differentiation of progenitor cells, we found that pure-oligodendrocyte were expressed 04 , which is labeled immature-oligodendrocyte and mature-oligodendrocytes. They also acquired the ability to bind $\mathrm{O1}$, but they were not expressed MBP on their surface. For their survival and differentiation, the cells require the addition of type1-astrocytes as a co-culture. Our results suggest that the stage-specific differentiation of oligodendrocytes into mature types does occur in a restricted manner, assisting with type1-astrocytes in culture.

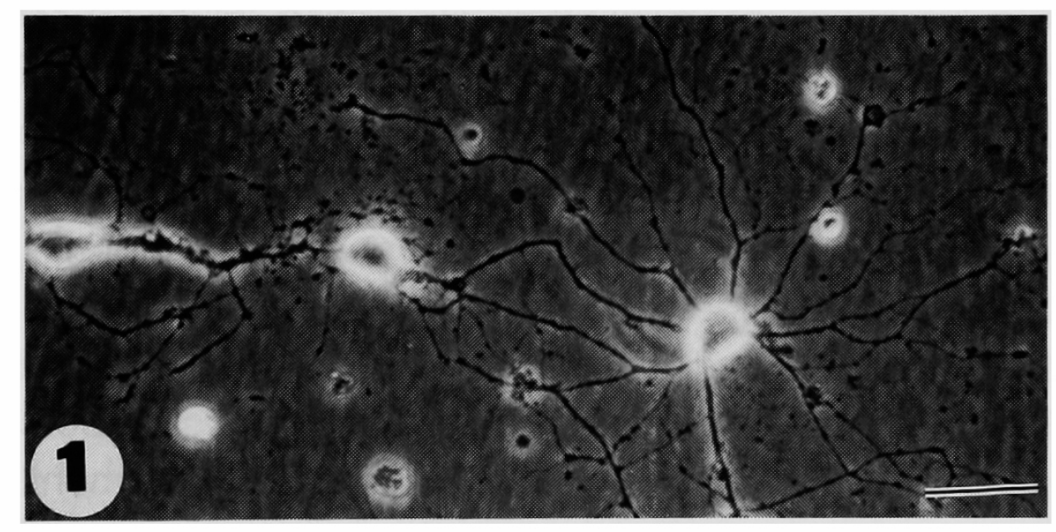

Fig.1

30-day-old stage-specific

immature oligodendrocytes

$$
\mathrm{Bar}=34 \mu \mathrm{m}
$$




\title{
Abstracts for Posters
}

\section{P29}

\section{The Connection between Granule Cells and Mossy Fibers Changes Dynamically during Postnatal Cerebellar Development.}

\author{
Miwako OZAKI, Tsutomu HASHIKAWA ${ }^{1}$ and Ryoji YANO \\ Lab. for Cellular Information Processing, ${ }^{1}$ Lab. for Brain Structure and Function, \\ Frontier Research Program, RIKEN, Saitama 351-01, JAPAN.
}

We are studying the mechanism of synaptic formation focusing on connection between cerebellar granule cells and mossy fibers on the cerebellar cortex. By using Dil labeling and wholemount immunostaining methods we have traced the events which were observed during synaptic maturation in developing cerebellar cortex. Dil labeling is a useful method in not only a study of process of axoplasmic transport but also a study of neural connections by anterograde and retrograde tracing (J. Neurosci. Methods, (1992) 42, 45-63). We have examined the axonal elongation process of mossy fibers and identified their target cells by Dil labeling. Furthermore target cells of mossy fibers were identified by wholemount immunostaining with antibodies against neurofilament, tenascin and adhesion molecule L1, as marker proteins for neurons, Bergmann glias and granule cells, respectively.

Although mossy fibers reached the cerebellar cortex in postnatal day1(P1), they did not seem to make contact even with postmigratory granule cells in internal granular layer (IGL) until P3. The number of granule cells stained by contact with mossy fibers increased gradually from P5 during development. As another interesting things, mossy fiber-purkinje cell interaction was observed in this developmental stage (P3 through P5). Following mossy fiber-purkinje cell interaction, mossy fiber-granule cell interaction in IGL was observed as main events at later stages. A large number of granule cells established contacts with mossy fibers in P7. Some mossy fibers went through molecular layer and extended to just inside edge of external granular layer (EGL), however these mossy fibers hardly entered into EGL. The thickness of stained mossy fibers was relatively large at early stages, gradually getting thinner during development. The mature form of glomeruli between mossy fibers and granule cells appeared from P14. The maturation of glomerulus proceeded from posterior lobule to anterior lobule. Moreover premigratory and migratory granule cells showed clusteringpattern stained by Dil in EGL and molecular layer. The stainning pattern might suggest that premigratory granule cells form functional modules by receiving signals from mossy fibers directly or indirectly during cerebellar development. 


\section{DIRECT OBSERVATIONS OF SYNAPTIC GLUTAMATE RELEASE MODULATED BY GABA AND NEUROTROPHINS IN CULTURED RAT CORTICAL NEURONS}

\section{Keiichi Torimitsu, *Ryoji Kurita and Osamu Niwa}

NTT Basic Research Laboratories, *NTT Advanced Technology, 3-1 MorinosatoWakamiya, Atsugi, Kanagawa 243-01

Glutamate, an excitatory neurotransmitter, is essential for synaptic transmission in brain nerve cells. Although various methods have been reported to measure the synaptic glutamate release, almost none of them can provide a continuous measurement becasue of its transience and small amount. Microdialysis-sampled liquid chromatography, which is commonly used in in vivo experiments, is not suitable for measuring such extremely small and fast transient phenomena. The use of labelled neurotransmitters requires a pretreatment and the estimation of the concentration. We developed, therefore, a novel glutamate detection method using glutamate oxidase and a glassy carbon electrode. In short, the method is based on the electrochemical reaction through HRP (horse radish peroxidase) insulated Os polyvinylpyridine. Using this method, we investigated the effect of neurotrophins on the synaptic glutamate release of cultured rat brain neurons.

Measurement was carried for cultured E18 rat cortical neurons and P2 rat hippocampal slices (300- $\mu \mathrm{m}$ thick). They were maintained in a DMEM-based culture medium containing insulin, heat-inactiated horse serum and heat-inactiated fetal bovine serum for 2 weeks at $37^{\circ} \mathrm{C}, 10 \% \mathrm{CO}$. NGF and BDNF were also added for slice preparation. A serum-free experimental medium (148 NaCl, $2.8 \mathrm{KCl}$, $2 \mathrm{MgCl} 2,2 \mathrm{CaCl} 2,10$ glucose in $10 \mathrm{Hepes}$ ) was used through the experiments. The solutions were continuously drawn by a CMA102 syringe pump at a rate of $6 \mu \mathrm{l} / \mathrm{min}$. They were introduced to a detector having a thin layer of glutamate oxidase together with the Os polyvinylpyridine on the carbon electrode through a glass capillary tube whose tip is few microns in diameter. The specificity of the detector to glutamate was confirmed using HPLC measurement with OPAMCE derivatization.

The dose dependence of the detector indicated a linear response through 10 $\mathrm{nM}$ to $10 \mu \mathrm{M}$. The addition of $100 \mathrm{mM} \mathrm{KCl}$ induced about $10-20 \mathrm{nM}$ glutamate release. As the release depended on the external calcium and was sensitive to TTX, it is thought to be synaptic. Inhibitory neurotransmitter GABA induced a large $\mathrm{Ca}$ - and TTX-sensitive transient increase of about $1 \mu \mathrm{M}$ in synaptic glutamate release, which was blocked by bicuculline, indicating this increase was produced through the GABAA receptor. The addition of neurotrophins, such as NGF and BDNF, induced either a transient or short continuous glutamate release. As these responses were calcium dependent and TTX sensitive, they are considered to be synaptic.

1. K. Torimitsu and O. Niwa and A. Kawana, Soc. Neurosci. 22, 75 (1996)

2. O. Niwa, K. Torimitsu, M. Morita et al., Anal. Chem. 68, 1865 (1996)

3. K. Torimitsu and O. Niwa, NeuroReport 8, 1353 (1997) 


\section{Abstracts for Posters}

P31

\section{REGION-SPECIFIC GENE EXPRESSION DURING THE EARLY FORMATION OF AXONAL TRACTS IN THE RAT FOREBRAIN}

\section{Yumiko Hatanaka}

Laboratory for Neuronal Recognition Molecules, Frontier Research Program, The Institute of Physical and Chemical Research, RIKEN. 2-1 Hirosawa, Wako, Saitama 351-01, Japan.

The forebrain is among the most complex regions of the mammalian central nervous system. Its large number of functionally and anatomically discrete regions are joined by specialized pathways. Because of its structural complexity, the mechanisms of forebrain development are poorly understood. For clarification of elemental structures and their development in the forebrain, I tried to isolate genes expressed in a region-specific manner, in the developing forebrain.

By use of a modified differential display method, two types of cDNAs, M1 and $M 2$, were isolated (1). Their expression profiles in the developing rat forebrain were analyzed in detail by in situ hybridization histochemistry. M1 expression was observed in the most ventral portion of the hippocampal rudiment with a sharp dorsal boundary, from embryonic day $(E) 12$ onward. Its location corresponded to the fimbrial anlage. As the fimbria developed, segregated expression of M1 and neuron-specific class III B-tubulin or GAP-43 became apparent, suggesting that a part of the neuroepithelium producing fimbrial neuroglia expresses M1 gene. M2 expression in the E12 telencephalon was confined to a part of the medial cerebral wall, including the presumptive preoptic region and hippocampus, with a diffuse dorsal boundary. At the same stage, M2 expression also occurred in a part of the dorsal diencephalon adjacent to the M2-positive telencephalic region, with a caudal extension along the dorsal midline, and in the zona limitans intrathalamica. M2 expression domains lacked neuron-specific class III B-tubulin immunoreactivity. As axonal tracts developed during later stages, M2 expression was found in association with the corpus callosum, hippocampal commissure, fimbria, optic nerve, stria medullaris, mammillothalamic tract and habenulopeduncular tract. In most cases, M2 expression was detected in corresponding regions prior to arrival of the earliest axons which could be detected by TAG-1- or GAP-43immunohistochemistry. These findings suggest the presence of specialized cell populations that express $\mathrm{M} 1$ and/or M2 genes along developing axonal pathways in the forebrain.

(1) Hatanaka, Y., Dev. Brain Res. $\underline{98}, 65-73$ (1997). 


\title{
Abstracts for Posters
}

P32

\section{Localization of $\mathrm{K}^{+}$channel complex on demyelinated axons.}

\author{
Hiroko Baba, Kensuke Nakahira, Tetsushi Kagawa, Yoshiro Inoue, and Kazuhiro \\ Ikenaka. \\ National Institute for Physiological Sciences, Myodaiji, Okazaki 444, Japan.
}

The transgenic mouse line, which is generated by overexpression of proteolipid protein gene (PLP-Tg) cause severe hypomyelination in homozygote and extensive demyelination in heterozygote (Kagawa et al, Neuron 1994). Histological analysis of heterozygote PLP-Tg showed that the CNS myelin structure was formed normally, then demyelination proceeded rapidly from 5 to 6 months after birth. By 7 months after birth, most of the axons in the spinal cord became naked. Since axonal degeneration is not significant in this PLP-Tg, this is a useful model animal to understand the functional significance of myelin on the CNS axons. In present study, heterozygous PLP-Tg was used to examine how the $\mathrm{K}^{+}$channel distribution was affected by demyelination at the molecular levels. Antibodies to various kinds of $\mathrm{K}^{+}$ channel subunits were kindly provided by Dr. Trimmer (SUNY, USA). In the spinal cord from wild type animals, $\mathrm{K}^{+}$channel $\beta$-subunit immunoreactivity was localized at paranodal regions where other $\alpha$-subunits such as $\mathrm{Kv1.1}$ and $\mathrm{Kv1} 1.2$ were located. The localization of $\mathrm{K}^{+}$channels on the axons in various ages of PLP-Tg were examined by double immunostaining technique using antibodies to $\beta$-subunit and myelin proteins. The spinal cord from 4.5-months-old PLP-Tg showed no significant demyelination and regular paranodal localization of $\beta$-subunit immunoreactivity. However, this characteristic immunoreactivity was changed in demyelinating spinal cord from older animals, suggesting reorganization of $\mathrm{K}^{+}$channels on demyelinated axons. A similar changes in the localization of $\mathrm{Na}^{+}$channels were previously reported. Therefore the dynamic reorganization of these channels occurs after chronic demyelination and it changes electric excitabilities. Taken together with the previous report of the channel organization in hypomyelinating mouse models, the presence of normal myelin sheath is required to maintain the specific channel localizations. 


\title{
Abstracts for Posters
}

\section{P33}

\section{REGULATION OF MICROTUBULE-ASSOCIATED PROTEIN MAP1B-TUBULIN INTERACTIONS BY ACIDIC PHOSPHOLIPIDS}

\author{
Emiko Yamauchi, Koiti Titani, Hisaaki Taniguchi \\ Division of Biomedical Polymer Science, Institute for Comprehensive Medical \\ Science, Fujita Health University, Toyoake, Aichi 470-11, Japan
}

Microtubule-associated protein MAP1B, a major neuronal cytoskeletal protein, is expressed highly during the early stage of brain development and is essential for the brain development. The protein is localized in the membranecytoskeletal fractions of growth cone, and is thought to play an important role in the microtubule-membrane interactions and/or in the regulation of the microtubules during the neurite extension. In the present study, we show that MAP1B purified from rat brain specifically binds to acidic phospholipids, and that the binding regulates the interaction of MAP1B with taxol-stabilized tubulin. Binding of MAP1B to phospholipid vesicles was first studied by sedimentation assay. When purified MAP1B was mixed with large unilamellar vesicles of phosphatidylserine, phosphatidylinositol, or phosphatidic acid, and subjected to centrifugation, similar bindings were observed with all the acidic phospholipids. On the contrary, no significant binding was observed when phosphatidylcholine (PC), a neutral phospholipid, was used. These results indicate that MAP1B has an intrinsic ability to bind to physiological membranes containing these acidic phospholipids. Next, the effects of phospholipids on the MAP1B-tubulin interaction were studied. Purified MAP1B co-sedimented with taxol-stabilized tubulin, demonstrating the ability of the protein to bind to the microtubules. The addition of PC to the mixture did not show any effect on the MAP1B-tubulin interaction. However, when acidic phospholipids were included in the mixture, MAP1B did not co-sediment with tubulin, but remained in the supernatant. The acidic phospholipids, therefore, can regulate the tubulin-MAP1B interactions. A $120 \mathrm{kDa}$ microtubule-binding fragment produced by chymotrypsin digestion behaved similarly to the intact protein. To test if the acidic phospholipids and tubulin share the same binding site in MAP1B, 3 peptides corresponding to the microtubule-binding domain were synthesized. One peptide was based on the $\mathrm{N}$-terminal half of the domain containing typical KKE repeats and the other on the C-terminal half of basic amphiphilic character. Only the peptides derived from the $C$-terminal half bound to acidic phospholipids. These results suggest that MAP1B binds to membranes through the C-terminal half of its tubulin binding domain, and the acidic phospholipids competes with tubulin for the same binding site. The phospholipid-dependent regulation of the MAP1B-tubulin interaction may be important for the dynamic regulation of the microtubules during the neurite extension. 


\section{Abstracts for Posters}

\section{P34 \\ MINIATURE SYNAPTIC CURRENTS IN NEURAL
SYNAPTOBREVIN-NULL DROSOPHILA MUTANTS}

Motojiro Yoshihara1 ${ }^{1}$ Atsushi Ueda1, Dawel Zhang1, David L. Deitcher?, Thomas L. Schwarz ${ }^{3}$ and Yoshiaki Kidokoro1

IInstitute for Behavioral Sciences, Gunma University School of Medicine, 3-39-22

Showa-machi, Maebashi 371, Japan. 2Section of Neurobiology and Behavior, W125 Mudd Hall, Cornell University, Ithaca, NY 14853, USA 3 Department of Molecular and Cellular Physiology, Stanford University Medical Center, Stanford, CA 94305-5426, USA

Synaptic transmission is caused by fusion of synaptic vesicles containing transmitter molecules to plasma membrane. The fusion is believed to be mediated by vesicle proteins (VAMP/synaptobrevin, synaptotagmin, etc.), plasma membrane proteins (syntaxin, SNAP-25, etc.), and cytoplasmic proteins ( $\alpha$-SNAP, NSF etc.). All these proteins have been identified in Drosophila. Thus, because of established powerful genetic techniques, the Drosophila neuromuscular synapse provides an ideal model system for studies of functions of the synaptic proteins

The SNARE hypothesis proposes docking of vesicles to occur via interaction between vesicle membrane protein, VAMP/synaptobrevin (V-SNARE) and plasma membrane protein, syntaxin (t-SNARE). In Drosophila, two synaptobrevin genes have been identified. One is a ubiquitous type, syb (1), and the other is neural type, $n$-syb (2). In Drosophila transformants in which $n$-syb product was cleaved by tetanus toxin, no nerve- evoked synaptic currents were observed but miniature synaptic currents (MSCs) remained (3). To further characterize synaptic transmission in the absence of neural synaptobrevin, we examined MSCs in homozygous embryos for a null mutation of $n$-syb. In the mutant stimulation did not evoke synaptic currents but MSCs were observed. The MSC frequency increased in high $\mathrm{K}^{+}$saline in the presence of external $\mathrm{Ca}^{2+}$. The relation between the MSC frequency and $\mathrm{Ca}^{2+}$ concentration shifted toward higher $\mathrm{Ca}^{2+}$ concentration compared with control animals. In the mutant the MSC frequency also increased with $\mathrm{Ca}^{2+}$ ionophore, A23187, and with black widow spider venom, but to the lesser extent compared with control for a given concentration of external $\mathrm{Ca}^{2+}$. Thus the $\mathrm{Ca}^{2+}$ sensing machinery for synaptic transmission might be less effective in the mutant. Tetanic stimulation induced asynchronous release of transmitter in the mutant. This asynchronous release occurred more frequently toward the end of tetanus and continued to occur after its termination. Furthermore, this did not happen in the absence of $\mathrm{Ca}^{2+}$, suggesting that voltage-gated $\mathrm{Ca}^{2+}$ channels are playing a role for this phenomenon. During asynchronous release occasionally synaptic currents occurred 4 to $10 \mathrm{~ms}$ after the onset of stimulus, which happened more often than expected for mere coincidence. Thus, in the mutant nerve stimulation could evoke synaptic currents in the facilitated state.

In n-syb-null mutant voltage-gated $\mathrm{Ca}^{2+}$ influx at the presynaptic terminal does occur, but the coupling between transient elevation of cytoplasmic $\mathrm{Ca}^{2+}$ during an action potential and vesicle fusion may be impaired.

(1)Chen et al. (1993). Gene 131, 175-181

(2)DiAntonio et al. (1995). J. of Neurosci. 13, 4924-4935

(3)Broadie et al. (1995). Neuron 15, 663-673 


\section{Abstracts for Posters}

\section{P35}

\section{Perpendicular contact guidance of CNS neurons; is it operative in the brain cortex development?}

Katsuhiko Ono', Isao Nagata ${ }^{2}$, Taro $\mathrm{Hama}^{3}$ and Norio Nakatsuji ${ }^{3}$ 'Department of Anatomy, Shimane Medical University, Izumo 693, ${ }^{2}$ Department of Cell Biology, Tokyo Metropolitan Institute for Neuroscience, Tokyo 183, and ${ }^{3}$ Mammalian Development Laboratory, National Institute of Genetics, Mishima 411, Japan.

We have reported that rodent CNS neuroblasts show parallel and perpendicular contact guidance on aligned neurite bundles in microexplant cultures of the cerebellar and cerebral cortices and other parts of the $\mathrm{CNS}^{1-3)}$. In contrast, such perpendicular orientation was not exhibited by the PNS neurons. Such perpendicular orientation of the CNS neurons occurs even on artificially fabricated parallel micro-structures on a quartz plate, indicating importance of the physical surface structure in the perpendicular guidance of neurons ${ }^{4)}$.

Perpendicularly oriented cells made many side-spikes extending from the leading neurite process, and they made close contact to parallel micro-structures of the substrate. The perpendicular guidance may play important roles in development of the CNS tissue architecture. Here, we present three groups of data that suggest operation of such unique guidance system in the brain cortex development.

(1) Morphology of the vertically migrating granule cells in the postnatal mouse cerebellum was examined in detail with Golgi method, laser scanning confocal microscopy, and transmission electron microscopy ${ }^{5}$. Our study indicated presence of many side-spikes extending horizontally from the vertically oriented leading cell process, and these side-spikes made direct contact to the horizontal parallel fibers.

(2) Ventricular cells were labeled with fluorescent dye (PKH26) in the organ or slice culture of the mouse cerebral cortex isolated from E17-19 fetuses. After allowing cell migration for one day, horizontally oriented neuroblasts were frequently observed not only in the intermediate zone but also in the cortical plate, which is made of radially (vertically) aligned bundles of the radial glia and neuroblasts, and in which there is no horizontal structure. In addition, these cells had many side-spikes extending vertically from the horizontal cell process.

(3) Injection of Dil microcrystals into the fetal ventricle and examination of the brain slices after a few days visualized many Dil-labeled neuroblasts after migration from the ventricular layer. There were horizontally oriented cells in the cortical plate as well as in the intermediate zone. The perpendicular contact guidance can explain horizontal orientation in the cortical plate, in which cells are surrounded by the vertical parallel structures.

1) Nakatsuji \& Nagata, Development 106, 411-447 (1989).

2) Nagata \& Nakatsuji, Devel. Brain Res. 52, 63-73 (1990).

3) Nagata \& Nakatsuji, Development 112, 581-590 (1991).

4) Nagata, Kawana \& Nakatsuji, Development 117, $401-408$ (1993).

5) Ono, Shokunbi, Nagata, Tokunaga, Yasui \& Nakatsuji, Exp. Brain Res., in press. 


\title{
Abstracts for Posters
}

P36

\section{Optical mapping of neural responses in the embryonic rat brainstem: early functional organization of vagal nuclei}

\author{
K. Sato, Y. Momose-Sato, Y. Arai and K. Kamino
}

Department of Physiology, Tokyo Medical and Dental University School of Medicine, 1-5-45 Yushima, Bunkyo-ku, Tokyo 113 , Japan

Multiple-site optical recording of transmembrane potential changes was employed to monitor the vagal response in the embryonic rat brainstem slice preparation. Slice preparations with vagus nerve fibers attached were dissected from 13 to 16 days old embryonic (E13-E16) rat brainstems, and they were stained with a voltage-sensitive merocyanine-rhodanine dye (NK2761). Electrical activities in response to vagal stimuli were optically recorded simultaneously from many sites using a 1020- or 128-element photodiode array measuring system. In the E13-E14 preparations, spike-like optical signals were recorded, and they were classified into two types of signal shapes: one was a narrow (about $15 \mathrm{msec}$ half width) signal (Type I), and the other was a broader (about $20 \mathrm{msec}$ half width) signal (Type I). Type l-signals were evoked in the dorsolateral area, and Type Isignals were recorded from the dorsomedial area ipsilateral to the stimulated side of the preparation. Comparison with the morphological view obtained from Dillabeling experiments implies that the Type I-signal response area corresponded to the nucleus of the tractus solitarius, and the Type I-signal response area, to the dorsal motor nucleus of the vagus nerve. In the E15-E16 preparations, Type Isignals were followed by a slow signal related to glutamate-mediated excitatory postsynaptic potentials in normal Ringer's solution. In contrast, in the E14 preparation, a small slow signal was evoked only in a $\mathrm{Mg}^{2+}$-free solution. These results reveal the initial event of synaptic transmission within the nucleus of the tractus solitarius of the rat embryo. In the E15 and E16 preparations, although the nucleus of the ambiguus is morphologically identified, no neural responserelated optical signal was observed. Further, we mapped the dynamic spatiotemporal patterns of the evoked optical signals, and traced the early phase of the functional organization of the vagus related cranial nuclei.

[References]

1) Komuro, H., Sakai, T., Momose-Sato, Y., Hirota, A. \& Kamino, K. (1991)

J. Physiol. (Lond) 442, 631-648.

2) Momose-Sato, Y., Sakai, T., Komuro, H., Hirota, A. \& Kamino, K. (1991)

J. Physiol. (Lond) 442, 649-668.

3) Momose-Sato, Y., Sakai, T., Hirota, A., Sato, K. \& Kamino, K. (1994)

J. Neurosci. 14, 7572-7584.

4) Sato, K., Momose-Sato, Y., Sakai, T., Hirota, A. \& Kamino, K. (1995) J. Neurosci. 15, 2123-2140.

5) Sato, K., Momose-Sato, Y., Hirota, A., Sakai, T. \& Kamino, K. (1996) Neuroscience 72, 833-846.

6) Momose-Sato, Y., Sato, K., Hirota, A., Sakai, T. \& Kamino, K. (1997) Neuroscience, in press. 


\title{
Abstracts for Posters
}

P37

\begin{abstract}
ANALYSIS OF THE REGULATORY MECHANISMS OF THE SYNAPTIC EXOCYTOSIS USING AN AUTAPSE IN THE CULTURED RAT HIPPOCAMPAL NEURON.
\end{abstract}

OSANAI M, TAKADA $M^{\star}$, FUJIWARA T, AKAGAWA K AND YAMAGUCHI K Department of Physiology, Kyorin University School of Medicine, 6-20-2 Shinkawa, Mitaka, Tokyo 118 JAPAN *Department of Physiology, Saitama Medical College, Moroyama, Saitama 350-04 JAPAN

To investigate the molecular mechanisms underlying the synaptic exocytosis and its regulation in the mammalian central synapse, an autapse, a synapse made by the neuron on itself in a micro-island culture provides a useful experimental system, since changes in both pre-and postsynaptic properties of the particular synapse elicited by some treatments can be detected by recording from one neuron, and which pre- or postsynaptic site is affected can be determined by the quantal analysis. Using this experimental system and the whole-cell patch clamp methods, we investigated the regulating mechanism of the synaptic exocytosis in the mammalian central neuron.

First, we analyzed effects of antibody against HPC-1/syntaxin 1A on the synaptic transmission. Intracellular application of antibody against HPC$1 /$ syntaxin $1 A$ through a whole-cell patch-pipette enhanced the amplitude of the autaptic excitatory postsynaptic current (epsc), though it did not change the asynchronous epsc amplitude, indicating that the antibody increased release of the neurotransmitter from the presynaptic terminal. This suggests a suppressive role for HPC-1/syntaxin $1 \mathrm{~A}$ in exocytosis at the mammalian central synapse.

Second, to elucidate the modulatory mechanism of the synaptic exocytosis, the dependence of epsc amplitude and paired pulse facilitation (ppt) on extracellular $\mathrm{Ca}^{2+}$ concentration $\left(\left[\mathrm{Ca}^{2+}\right]_{\theta}\right)$ were analyzed using the autapse of the cultured rat dentate gyrus neuron. The $\left[\mathrm{Ca}^{2+}\right]_{\theta}$ dependency of the epsc amplitude was well described by the multiple binding model with $n$ (Hill coefficient) $=3.7$ and $K_{m}=0.97 \mathrm{mM}$. The $\left[\mathrm{Ca}^{2+}\right]_{\mathrm{e}}$ dependency of $p p f$ was also described by modifying this model. This model also suggested that ppf decreased as the release probability $(p)$ increased. Using these models, we analyzed the mechanism of epsc-enhancement and concomitant ppf-reduction by forskolin. Analysis suggested that forskolin increased the release probability. 


\section{Abstracts for Posters}

P38

\section{SYNAPHIN/COMPLEXIN IS ESSENTIAL FOR SYNAPTIC TRANSMITTER RELEASE}

H. Tokumaru, L. L. Pelligrini, T. Ishizuka, K. Umayahara, H. Betz, T. Abe and G. J. Augustine Duke University, Durham, NC 27710, Max-Planck Institute for Brain Research, Frankfurt 71, Brain Research Institute, Niigata University, Niigata 951 Japan and Marine Biological Laboratory, Woods Hole, MA 02543

Synaphin (also known as complexin) is a cytosolic protein that competes with $\alpha$-SNAP for binding to syntaxin in vitro. These binding interactions suggest that synaphin regulates SNARE complexes, which are known to be important for synaptic transmission. We have studied the in vivo role of synaphin in neurotransmitter release by using the giant synapse of the squid, Loligo pealei. Microinjection of recombinant synaphin into the giant presynaptic terminal caused a strong inhibition of transmitter release, indicating a negative role for synaphin in transmitter release. To further probe the function of squid synaphin, we isolated synaphin cDNA from a squid optic lobe cDNA library. A full-length cDNA encoding squid synaphin was found and predicted a protein with about $40 \%$ sequence identity to mammalian synaphin. Sequence identity is particularly high in the central, syntaxin-binding domain of these homologues. We designed two synthetic peptides from this domain, one of which prevented the in vitro binding of syntaxin to synaphin but not to $\alpha$-SNAP. Microinjection of the effective peptide into squid giant presynaptic terminals caused a rapid, complete and reversible inhibition of evoked transmitter release without affecting presynaptic calcium signaling. In contrast, control peptides containing the same amino acids in a scrambled order had no effect on release, indicating that synaphin binding to syntaxin also has a positive role in transmitter release. From these data, we conclude that binding of synaphin to syntaxin initially prevents SNARE complex formation but subsequently facilitates the molecular interactions that lead to fusion of synaptic vesicles. Supported by NIH and HFSP grants. 


\title{
Central respiratory chemoreception in the developing rat brainstem: dynamic imaging analysis with fluorescent voltage sensitive dye
}

\author{
Okada, Y. ${ }^{1,2}$, Chen, $Z_{.}^{2}$ and Eldridge, F.L. ${ }^{2}$ \\ ${ }^{\mathrm{I}}$ Department of Medicine, Keio University Tsukigase Rehabilitation Center \\ Amagiyugashima 410-32 Japan \\ ${ }^{2}$ Department of Physiology, University of North Carolina, Chapel Hill NC 27599 USA
}

Regional and cellular specification of the central respiratory chemosensing mechanism in the mammalian brainstem have not been defined. We have applied an optical recording technique to analyze the dynamic process of central respiratory chemoreception in the developing rat bainstem. The brainstem was isolated from the neonatal rat (Sprague-Dawley; $1-5$ days old) ${ }^{1}$ ), stained with fluorescent voltage sensitive dye (RH-795), and continuously superfused with artificial cerebrospinal fluid (aCSF) in a recording chamber. Responses of cellular membrane potentials to $\mathrm{CO}_{2}$ changes on the ventral medullary and transversely sectioned surfaces were analyzed as dynamic images with a high sensitivity optical recording system (HR Deltaron 1700, Fuji Film, Tokyo) ${ }^{2}$ ). Regions with cellular depolarization were found in the ventral (midline, parapyramidal and ventrolateral) medulla, and the distribution of the $\mathrm{CO}_{2}$-depolarized regions well corresponded with that of surface vessels. Propagation of the excitatory signals from the ventral medullary surface to a deeper region was dynamically visualized on a transverse section. With synaptic blockade $\left(\mathrm{Cd}^{2+}\right.$, TTX or low $\mathrm{Ca}^{2+}$-high $\left.\mathrm{Mg}^{2+}\right)$ aCSFs, cellular depolarization was not found except for the surface layer (marginal glial layer), and the ventrodorsal excitation propagation was abolished. It is suggested that intrinsic $\mathrm{CO}_{2}$-excitatory regions are located in the surface layer of the ventral medulla, and that the excitation is synaptically transmitted to deep medullary regions. We propose a model of cellular architecture in central chemoreception on the basis of the above and our previous experiments ${ }^{3,4)}$ : primary chemoreceptor cells are located in the marginal glial layer adjacent to surface vessels and interneurons in the subsurface layer amplify and transmit the chemical information to the respiratory neuronal network.

\section{References}

1) Okada, Y., Mückenhoff K., Holtermann G., Acker H. and Scheid P. (1993): Depth profiles of pH and $\mathrm{PO}_{2}$ in the isolated brain stem-spinal cord of the neonatal rat. Respir. Physiol., 93:315-326

2) Iijima. T., Witter, M.P., Ichikawa, M., Tominaga, T., Kajiwara, R. and Matsumoto, G. (1996):

Entorhinal-hippocampal interactions revealed by real-time imaging. Science, 272:1176-1179

3) Okada, Y., Chen, Z. and Eldridge, F.L. (1996): Sites of the central respiratory chemoreception in the rat medulla investigated by microinjection of $\mathrm{CO}_{2}$-enriched mock cerebrospinal fluid (CSF). FASEB J., 10:A643

4) Chen, Z., Okada, Y. and Eldridge, F.L. (1997): Hypercapnia-induced c-fos expression in cholinergic cells within the marginal glial layer of the rat ventral medulla oblongata. FASEB J., 11 : A131 


\section{Abstracts for Posters}

\section{P40}

\section{REGULATION OF GABAERGIC CELL-SPECIFIC GENE TRANSCRIPTION}

Y. Yanagawa ${ }^{1}$, T. Kobayashi ${ }^{1}$, K. Ishii ${ }^{1}$, M. Kutsuwa ${ }^{1}$, H. Sakagami ${ }^{2}$, H. Kondo ${ }^{2}$, F. Tashiro ${ }^{3}$, J. Miyazaki ${ }^{3}$, S. Tamura ${ }^{1}$

${ }^{1}$ Institute of Development, Aging and Cancer, ${ }^{2}$ School of Medicine, Tohoku University, Sendai, 980, Japan; ${ }^{3}$ School of Medicine, Osaka University, Suita, 565, Japan

The choice of a specific complement of neurotransmitter is a crucial step in the aquisition of a differentiated phenotype by developing neurons. The molecular mechanisms governing the aquisition and maintenance of neurotransmitter phenotypes are only beginning to be understood, as more regulatory sequences and transcription factors affecting pathways of neuronal differentiation are being characterized. $\gamma$-Aminobutyric acid (GABA), one of the major neurotransmitters in the nervous system, is synthesized by glutamic acid decarboxylase (EC 4.1.1.15; GAD), currently the most specific marker for GABAergic neurons.

GAD has been shown to exist in two forms encoded by separate genes in mammals. These forms have been denoted GAD65 and GAD67 respectively, the subscript numbers referring to their molecular weight. In order to elucidate the regulatory mechanisms of mouse GAD (mGAD) gene expression and to define the promoter regions that are essential for its GABAergic cell-specific expression, we have isolated the 5 '-flanking region of the mGAD genes. Sequence analysis of the $5^{\prime}$-flanking region of the mGAD67 gene revealed the presence of numerous neuron-specific cis regulatory elements such as the neuron-restrictive silencer element and the CCAGGAG motif. In transgenic mice, we have examined the expression pattern conferred by a $10 \mathrm{~kb}$ fragment of the mGAD67 gene upstream region fused to the lacZ reporter gene. The transgene expression was observed in neurons of particular brain regions such as cerebral cortex and basal ganglia, where expression of GAD67 gene has been reported to be abundant. These results suggest that the $10 \mathrm{~kb}$ fragment may contain cisacting elements essential for its GABAergic cell-specific expression in the brain. 